\title{
RIGID TRACKING FOR SCALE AND ROTATION VARYING TARGETS FROM MOVING CAMERA
}

\author{
Karthika Pragadeeswari $\mathbf{C}^{1} *$ and Yamuna $\mathbf{G}^{2}$ \\ ${ }^{1}$ Department of ECE, Alagappa Chettiar Government College of Engineering and Technology, \\ Karaikudi, India 630003(e-mail: bk.karthika1969@gmail.com) \\ ${ }^{2}$ Department of ECE, FEAT, Annamalai University, Chidambaram, India (e-mail: \\ yamuna.sky@gmail.com) \\ * Corresponding author
}

\begin{abstract}
Targets when move rapidly needed to be tracked in many significant fields such as in combat applications. Objects undergoes many scale changes and also undergoes rotation variance. The target when viewed from static position, the size becomes smaller as the target moves farther and farther. Tracking the targets needs more attention and this can be done by Improved optical flow to which feature extraction through Histogram of Oriented Gradients and Random Sample Consensus (RANSAC) algorithm for scale and rotation invariance is added. The performance of the method is measured by its computation time, accuracy and high true positive values and other related parameters simulated in MAT LAB.
\end{abstract}

Index Terms: Extraction, Tracking, Human, scale and rotation invariance, Moving camera.

\section{INTRODUCTION}

Surveillance can be done by both static and moving cameras. Whenever video taken from a static camera is used, the dense optical flow itself is sufficient to determine the moving object in the scenes. However, in a [1] nonstatic camera, all pixels are moving making which lead to incapability of optical flow to differentiate the moving objects from the static background. In order to solve this problem, a stabilization method is incorporated by the mean of global motion extraction, which can be done by analysing the homography transformation between two consecutive frames. Finally, by applying a threshold on the dense optical flow, the region of moving object is acquired. [2] study presents a system using depth information and visual odometry to detect moving object. It also adopts an adaptive thresholding method to enhance performance on detection. The proposed system can accurately detect moving objects and produce good results.

In the proposed framework moving object detection and tracking is taken care with the PC

Copyright $($ C) Author vision methods called an optical flow. The global positioning framework works in indoor and outside situations with better exactness by utilizing this method. This methodology figures the movement between two images outlines which are taken at various time spans in the video. The optical stream depicts the motion and time pixels in subsequent frames. The movement vector gives exact movement assessment of an object in the progressive frames of the video. The obtained video outlines are separated by utilizing median filter. This eliminates the common segment included during preprocessing. This makes the proposed framework powerful in noise. It likewise raises the general efficiency of tracker. Because of this, it draws considerations of a few scientists, foundations and business associations. To build up a strong framework with ongoing moving object recognition, characterization and following abilities is our principle motivation in considering this issue. 


\section{RELATED WORK}

Item Tracking utilizing SIFT and Kalman Filter [3] characterizes an article following depends on SIFT (Scale-Invariant Feature Transform) and Kalman channel. The SIFT calculation identifies the invariant component focuses which are utilized in distinguishing the objective item in non-impeded condition. In the event that the SIFT calculation neglects to follow the article if there should be an occurrence of impediment, Kalman channel which can foresee the objective area is utilized at such cases to decide the area of the objective. An Object Tracking System Based on SIFT and SURF Feature Extraction Methods [4] portrays an article recognition and global positioning framework which depends on SIFT and SURF include extraction strategies. From the assessment results, we saw that the precision of coordinated central issues of SURF calculation are higher than SIFT.

SURF Tracking [5] portrays a generative model is proposed to portray the connection between nearby component movements and item worldwide movement. Investigations show that our system is adaptable and hearty in managing appearance changes, foundation mess, enlightenment changes and impediment. Item Recognition and Tracking dependent on Object Feature Extracting [6] depicts a CAMSHIFT is a typical calculation for following an intrigue object for ongoing situations. In any case, the calculation utilizes just shading highlights, so it isn't powerful to the general condition and brightening. This strategy has the issue of losing the intrigue target when a comparable shading exists in the foundation since it is touchy to brightening and commotion. This issue can be unravelled by the incorporated technique which perceives the objective article utilizing SURF for include extraction and can follow the item by CAMSHIFT.

Article Tracking Method Based on SURF [7] depicts an intrigue focuses are distinguished by SURF highlight descriptor and the technique shows improvement continuously following vigor against appearance varieties, scale changes and jumbled scenes. A Comparative Study of CF, LBP, HOG, SIFT, SURF and BRIEF for Security and Face Recognition [8] portrays a different component descriptor and looking at the calculation time and the outcomes shows that histogram of situated angles creates more precise and less calculation time. Among this overview HOG gives best exactness diminished calculation time. Histograms of Oriented Gradients [9] Learning a Features for Object Detection, Simplified Gradient, Cells, Contrast Normalization, Contrast Normalization Details, execution of HOG.

Since 1981 RANSAC has become a major device in the PC vision and picture preparing network. In 2006, for the 25th commemoration of the calculation, a workshop was sorted out at the International Conference on Computer Vision and Pattern Recognition (CVPR) to sum up the latest commitments and varieties to the first calculation, generally intended to improve the speed of the calculation, the strength and exactness of the assessed arrangement and to diminish the reliance from client characterized constants. RANSAC can be delicate to the decision of the right commotion edge that characterizes which information focuses fit a model launched with a specific arrangement of boundaries. In the event that such edge is excessively huge, at that point all the theories will in general be positioned similarly (acceptable).

\section{Proposed Methodology}

Input video captured from dynamic camera or drone is considered. Pre-processing involves conversion of input video into frames. Then, the redundant frames have to be identified. Remove the redundant frames. This helps for further processing which is explained in the following sections as shown in Fig.1.

\section{A. Object Detection}

Pretty much every video observation framework manages movement and article location. The motivation behind movement 
identification is dividing districts pertinent to moving items from the excess of a picture. When movement and article are identified, observation frameworks track moving items from one edge to next in a picture grouping. The calculations utilized for movement recognition normally have crossing point with movement identification during handling.

\section{B. Object Detection}

Recognition and investigation of movement patterns is accomplished for conduct comprehension and results in significant level depiction of activities and communications between the objects. Among the many object acknowledgment strategies like Scale Feature Invariant Transform, Speed Up Robust Feature, Binary Robust Independent Elementary Features, Local Binary Pattern, Histogram of Oriented Gradients (HOG) assumes a proficient function for object acknowledgment. HoG descriptors for evaluating and speaking to both shape and surface. HoG descriptors are fundamentally used to depict the auxiliary shape and presence of an object in an image, making them astounding descriptors for object classification. Feature extraction is done by computing intensity gradients for all the pixels in the frame. Then, bring gamma and color to its normal state by the process of normalisation. Then, compute weights in each cell and normalise contrast. Collect $\mathrm{HoG}$ that represents the extracted features.

\section{RANSAC Algorithm}

Random sample consensus (RANSAC) is an iterative technique to appraise boundaries of a numerical model from a lot of observed information that contains outliers when anomalies are to be concurred no impact on the estimations of the values. Therefore, it also can be interpreted as an outlier detection method [10]. It is a non-deterministic algorithm as in it creates a sensible outcome just with a specific likelihood, with this likelihood expanding as more cycles are permitted. RANSAC is utilized to take care of the Location Determination Problem (LDP), where the objective is to decide the focuses in the space that venture onto a picture into a lot of landmarks with known areas. A fundamental supposition that will be that the information comprises of "inliers", i.e., information whose dispersion can be clarified by some arrangement of model boundaries, however might be liable to commotion, and "exceptions" which are information that don't fit the model. The anomalies can come, for instance, from outrageous estimations of the clamor or from mistaken estimations or off base theories about the understanding of information. RANSAC likewise expect that, given a (generally little) arrangement of inliers, there exists a system which can assess the parameters of a model that ideally clarifies or fits this information. An advantage of RANSAC is its ability to do robust estimation [11] of the model parameters, i.e., it can estimate the parameters with a high degree of accuracy even when a significant number of outliers are present in the data set. 


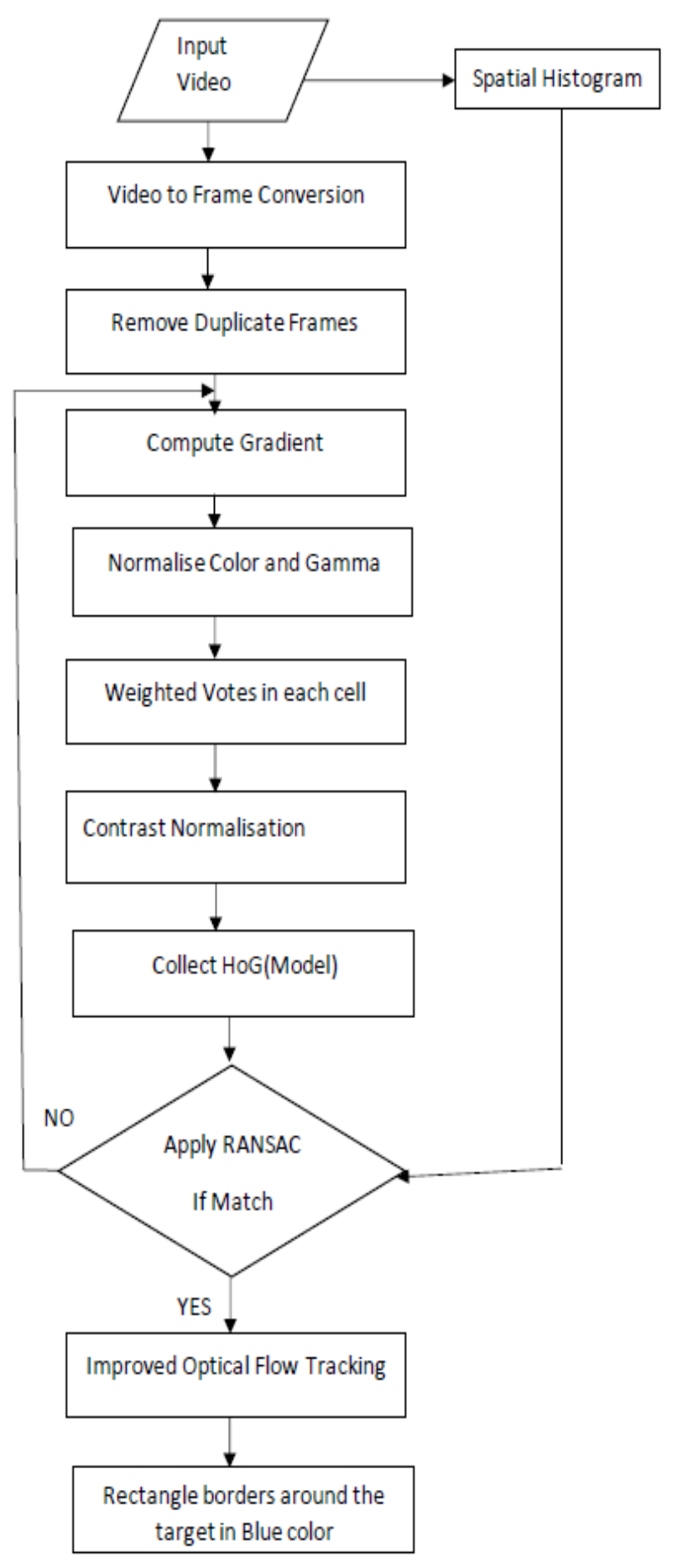

Figure 1. Block Diagram of Implemented Method.

\section{Object Tracking}

Optical flow is a vector-based methodology that assessments movement in video by coordinating focuses on objects over picture frame(s). Under the assumption of brightness steadiness and spatial perfection, optical stream is utilized to depict the coherent motion of points or highlights between picture frames. Optical stream-based motion segmentation utilizes attributes of flow vectors of moving items after some time to identify moving locales in a picture sequence. One key advantage of utilizing optical stream is that it is hearty to different and synchronous cameras and object motions, making it ideal for swarm examination and conditions that contain thick movement. Optical stream-based techniques can be utilized to identify autonomously moving articles even within the sight of camera movement. Aside from their weakness to picture noise, shading and non-uniform lighting, the majority of stream calculation techniques have huge computational necessities and are delicate to movement discontinuities. Ongoing execution of optical flow will regularly require a specialised hardware because of the unpredictability of the algorithm and modestly high frame rate for accurate measurements.

\section{RESULTS AND DISCUSSION}

We actualize our calculation with the OpenCV library. On Pentium-4 3.0GHZ machine, moderately, the calculation time is under 120ms for single casing of size 640 x 480 . We exhibit the presentation of our calculations through various video arrangements, and get promising outcomes.

Scale and Rotation Invariant is one of the tough challenges in object recognition. In scale invariant, the size of the targeted object continuously changes from larger to smaller, and vice versa. Rotation invariant leads to the objects can be rotated from left to right or right to left with so many angles. This proposed algorithm works efficiently in both the cases and gives good results. Various parameters also estimated. For airplane struck video which is shooted from a moving camera is real time is tested and the results are taken. It is cut so that it contains 1229 frames. The computation time is also reduced which is much less than traditional methods [12] are taken with elapsed 
time as 0.260308 milli seconds.

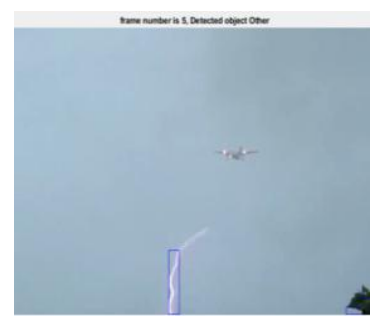

(a)

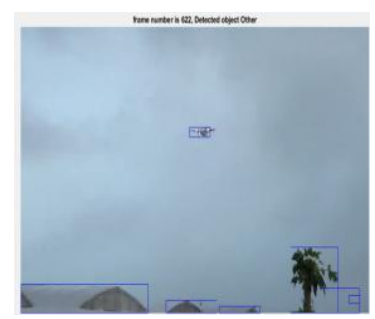

(c)

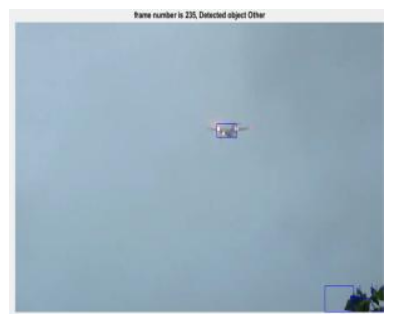

(b)

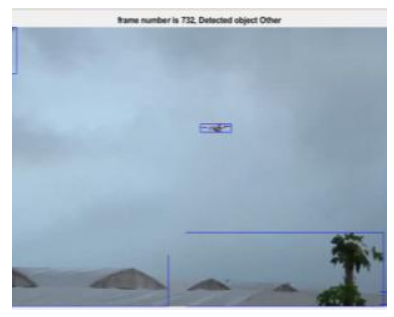

(d)
Figure 2. Scale and Rotation Invariant Detection Using HOG and RANSAC. (a) Frame No.5. (b) Frame No.235. (c) Frame No.622. (d) Frame No.732.

Fig. 2 depicts the different frame numbers such as 5, 235,622,732, are taken and the recognition as other not human is displayed in the result. The human features are the region of Interest. The tracking boundary output is shown in blue rectangular boxes. The accuracy of the tracker is shown from the parameters accuracy, True positive, True negative, False positive, false negative, sensitivity, specificity, precision, recall, F-measure, False Acceptance Rate and False Recognition Rate. The command window results are shown in Fig.3 (a)and (b) which shows the HoG results as other object not human and various parameter values are obtained respectively.

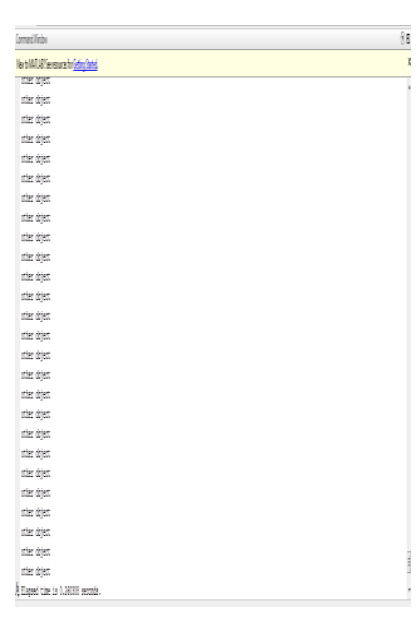

(a)

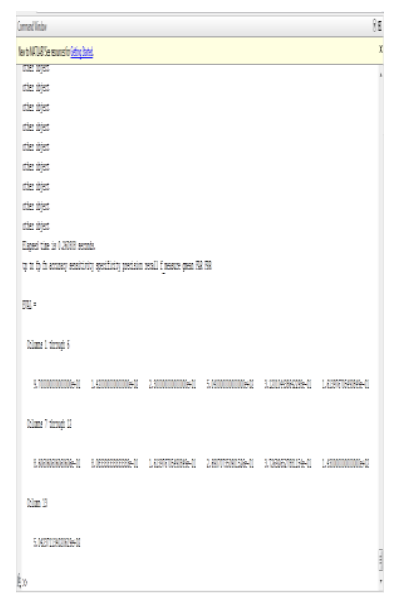

(b)
Figure 3. Command Window Result. (a) Recognition output as Human /Human /other object. (b) Parameter value at output.

(Table 1; Fig. 3(b)) represents the same values obtained as a result of implementation. Table 1 shows the unit less numbers which are depends on the number of frames and are nearing the ideal values. This execution achieves high true positive and low false negative values. Accuracy is $31 \%$ and precision is $80 \%$. False recognition ratio is almost 504 .

Table 1. Parameters values at output.

\begin{tabular}{lc}
\hline Parameters & Values \\
\hline True Positive & 504 \\
True Negative & 142 \\
False Positive & 23 \\
False Negative & 97 \\
Accuracy & 0.3120 \\
Sensitivity & 0.1614 \\
Specificity & 0.8606 \\
Precision & 0.8083 \\
Recall & 0.1614 \\
F-Measure & 0.2691 \\
False Acceptance rate & 143 \\
False Recognition rate & 504.23 \\
\hline
\end{tabular}

\section{CONClusion}

The method works well and tested with the real time video. The parameters related to this technique are accuracy, True positive, True 
negative, False positive, false negative, sensitivity, specificity, precision, recall, Fmeasure, False Acceptance Rate and False Recognition Rate are calculated from standard formulas. The scale and rotation variant moving targets are tracked with good accuracy. This result so obtained is because of the use of HoG for object recognition and RANSAC algorithm for scale and rotation invariance is added to improved optical flow method. The computation time is also greatly reduced which is the main drawback in literature and results are shown. In future, Deep learning techniques can be added to this method to recognize strongly with different objects.

\section{REFERENCES}

[1] Laksono Kurnianggoro, Ajmal Shahbaz, and Kang-Hyun Jo, "Dense Optical Flow in Stabilized Scenes for Moving Object Detection from a Moving Camera" in Proc. 16th International Conference on Control, Automation and Systems (ICCAS 2016) in HICO, Gyeongju, Korea Oct. 16-19, 2016.

[2] Sheng-Fuu Lin, Sheng-Huei Huang, "Moving Object Detection from a Moving Stereo Camera via Depth Information and Visual Odometry" in Proc. of IEEE Int. Conf. on Applied System Innovation IEEE ICASI, ISBN 978-1-5386-4342-6 437, 2018.

[3] Hemalatha B, Grevas Timi L, Krishnaamirthalaxmi V S," Object Tracking using SIFT and Kalman Filter", Int. J. of Scientific \& Engineering Research, vol. 9, no. 3, pp.197-202, March 2018.

[4] Yuki Sakai, Tetsuya Oda, Makoto Ikedaand Leonard Barolli," An Object Tracking System Based on SIFT and SURF Feature Extraction Methods", in Proc.18th Int. Conf. on Network-Based Information Syst., IEEE 2015.

[5] Wei He, Takayoshi Yamashita, Hongtao Lu, and Shihong Lao," SURF Tracking", in Proc.12th Int. Conf. on Computer Vision (ICCV), IEEE 2009.
[6] Yong-Hwan Lee, Hyochang Ahn , HanJin Cho , and June-Hwan Lee," Object Recognition and Tracking based on Object Feature Extracting", J. of Internet Services and Information Security (JISIS), vol.5, no. 3, pp. 48-57, August 2015.

[7] $\mathrm{Hu}$ shou, Wu Na,song huagun," Object Tracking Method Based on SURF", in Proc. Conference on Modelling and identity control, Americal Applied Science and Research Institute (AASRI) 2012.

[8] Kortli, Yassin, Jridi, Maher, Al Falou, Ayman, Atri, Mohamed," A Comparative Study of CFs, LBP, HOG, SIFT, SURF, and BRIEF Techniques for Face Recognition", Proc. of SPIE, 27 January 2020.

[9] Salma hamdi, Naeem Nisar sheikh," Object Detection using Histogram of Oriented Gradients", Capstone report, School of Science \& Engineering, Alakhawayn university, Spring 2018.

[10] T. Strutz "Data Fitting and Uncertainty", Springer Vieweg 2nd ed. 2016.

[11] Peter. J. Huber, Robust Statistics, Wiley, 1981, republished in paperback, 2004.

[12] Simon Denman, Vinod Chandran, Sridha Sridharan "Adaptive Optical Flow for Person Tracking", Proc. of the Digital Imaging Computing: Techniques and Applications (DICTA 2005), IEEE 07695-2467-2/05. 